\title{
The effect of twisted magnetic field on the resonant absorption of MHD waves in coronal loops
}

\author{
K. Karami ${ }^{1 *}$, K. Bahari ${ }^{2}$ \\ ${ }^{1}$ Department of Physics, University of Kurdistan, Pasdaran Street, Sanandaj, Iran \\ ${ }^{2}$ Institute for Advanced Studies in Basic Sciences (IASBS), Gava Zang, Zanjan, Iran
}

November 13, 2018

\begin{abstract}
The standing quasi modes in a cylindrical incompressible flux tube with magnetic twist that undergoes a radial density structuring is considered in ideal magnetohydrodynamics (MHD). The radial structuring is assumed to be a linearly varying density profile. Using the relevant connection formulae, the dispersion relation for the MHD waves is derived and solved numerically to obtain both the frequencies and damping rates of the fundamental and first-overtone modes of both the kink $(m=1)$ and fluting $(m=2,3)$ waves. It was found that a magnetic twist will increase the frequencies, damping rates and the ratio of the oscillation frequency to the damping rate of these modes. The period ratio $P_{1} / P_{2}$ of the fundamental and its first-overtone surface waves for kink $(m=1)$ and fluting $(m=2,3)$ modes is lower than 2 (the value for an untwisted loop) in the presence of twisted magnetic field. For the kink modes, particularly, the magnetic twists $B_{\phi} / B_{z}=0.0065$ and 0.0255 can achieve deviations from 2 of the same order of magnitude as in the observations. Furthermore, for the fundamental kink body waves, the frequency bandwidth increases with increasing the magnetic twist.
\end{abstract}

Keywords: Sun: corona - Sun: magnetic fields - Sun: oscillations

*E-mail: KKarami@uok.ac.ir 


\section{Introduction}

Transverse oscillations of coronal loops were first identified by Aschwanden et al. (1999) and Nakariakov et al. (1999) using the observations of Transition Region and Coronal Explorer (TRACE). Nakariakov et al. (1999) reported the detection of spatial oscillations in five coronal loops with periods ranging from 258 to $320 \mathrm{~s}$. The decay time was $14.5 \pm 2.7 \mathrm{~min}$ for an oscillation of $3.9 \pm 0.13 \mathrm{mHz}$. Also Wang and Solanki (2004) described a loop oscillation observed on 17 April 2002 by TRACE in $195 \AA$. They interpreted the observed loop motion as a vertical oscillation, with a period of $3.9 \mathrm{~min}$ and a decay time of $11.9 \mathrm{~min}$. Nakariakov et al. (1999) obtained that the amplitude of the oscillations decreases by more than 50 percent in several oscillation periods. To estimate the energy flux of the EUV kink oscillation observed by Nakariakov et al. (1999), we use the maximum kinetic energy flux of an oscillating loop given by $\frac{1}{2}\left(\pi R^{2} L\right) \rho v_{\max }^{2} /(2 \pi R L) \tau_{\mathrm{D}}=$ $\frac{1}{4} R \rho v_{\max }^{2} / \tau_{\mathrm{D}}$. Where $\rho, v_{\max }, R, L$ and $\tau_{\mathrm{D}}$ are the mass density, the peak loop velocity, the loop radius, the loop length, and the damping time of oscillation, respectively. Taking $R=2 \times 10^{3}$ $\mathrm{km}, \rho=2 \times 10^{-14} \mathrm{gr} \mathrm{cm}^{-3}$ for a typical coronal loop in active region and using $v_{\max }=47 \mathrm{~km}$ $\mathrm{s}^{-1}$ and $\tau_{\mathrm{D}}=14.5$ min given by Nakariakov et al. (1999), we obtain the energy flux as $2 \times 10^{4}$ erg $\mathrm{cm}^{-2} \mathrm{~s}^{-1}$ which is three orders of magnitude smaller than the radiated energy flux $10^{7}$ erg $\mathrm{cm}^{-2} \mathrm{~s}^{-1}$ in active regions (see Klimchuk 2006). Therefore, the energy flux in the EUV kink oscillations is unlikely to be sufficient to cover heating of coronal loops.

Since the discovery of the coronal green line during the 1869 eclipse which identified as Fe XIV spectral line by Grotrian (1939), different theories of coronal heating have been put forward and debated (for reviews see, e.g., Hollweg 1991: Narain and Ulmschneider 1996; Walsh and Ireland 2003; Erdélyi 2004, 2005; Klimchuk 2006; Erdélyi and Ballai 2007; Taroyan and Erdélyi 2009). Ionson (1978) was first to suggest that the resonant absorption of MHD waves in coronal plasmas could be a primary mechanism in coronal heating. Since then, much analytical and numerical work has been done on the subject. Rae and Roberts (1982) investigated both eikonal and differential equation approaches for the propagation of MHD waves in inhomogeneous plasmas. Hollweg (1987a,b) considered a dissipative layer of planar geometry to study the resonant absorption of coronal loops. Davila (1987) derived the resonant heating rate in the low-beta compressible resistive MHD approximation with the shear viscosity and found that the heating does not depend explicitly on the dissipation coefficients. Poedts, Goossens, and Kerner $(1989,1990)$ developed a finite element code to elaborate on the resonant absorption of Alfvén waves in circular cylinders.

Sakurai, Goossens, and Hollweg (1991a,b), Goossens, Hollweg, and Sakurai (1992), Steinolfson and Davila (1993) did much work on resonant absorption. Ofman, Davila, and Steinolfson (1994), and Erdélyi and Goossens (1994) included viscous and resistive dissipations in their analysis and concluded that the heating rate due to shear viscosity is comparable in magnitude to the resistive resonant heating. Also, they concluded that the heating caused by compressive viscosity is negligible. Goossens, Ruderman, and Hollweg (1995) extended the analysis of Sakurai et al. (1991b) on resonant Alfvén waves in nonuniform magnetic flux tubes for a static MHD equilibrium. They showed that the conservation law found in ideal MHD is valid in dissipative MHD. They derived analytical solutions to the dissipative MHD equations for the Lagrangian displacement and for the total pressure perturbation. Erdélyi and Goossens (1995) studied the heating of solar coronal loops by resonant absorption of Alfvén waves in visco-resistive MHD. They pointed out that under solar coronal conditions the two dissipative mechanisms are both operational and that electrical resistivity appears to be slightly more important than viscosity. Erdélyi and Goossens (1996) studied the effect of an equilibrium flow on resonant absorption of linear MHD waves in compressible viscous cylindrical magnetic flux tubes. They showed that 
the presence of the equilibrium flow is very determinant for resonant absorption and significantly affect the resonance absorption rate suffered by the incoming driving waves. Goossens, Andries, and Aschwanden (2002) used the TRACE data of Ofman and Aschwanden (2002) to infer the width of the inhomogeneous layer for 11 coronal loops. Ruderman and Roberts (2002) solved the initial value problem for a zero-beta loop driven by a kink perturbation. They pointed out that the observed damping rate of the coronal oscillations is due to the resonant damping of the quasi-mode kink oscillations. They estimated the inhomogeneity length scale (thickness of the boundary layer) of the loop with the data of Nakariakov et al. (1999). Note that Nakariakov et al. (1999) showed that the damping rate of the global kink oscillation observed by the TRACE can be justified by the energy absorption due to anomalous viscosity. Whereas Ruderman and Roberts (2002) cleared that for large values of the viscous Reynolds number, the damping rate is independent of the viscous Reynolds number and proportional to the ratio of the thickness of the inhomogeneous layer and the radius of the tube. Van Doorsselaere et al. (2004a) used the LEDA code (Large-scale Eigenvalue solver for the Dissipative Alfvén spectrum) to study the resistive absorption of the kink modes of cylindrical models. They concluded that, when the width of the nonuniform layer was increased, their numerical results differed by as much as 25 per cent from those obtained with the analytical approximation.

Safari et al. (2006) studied the resonant absorption of MHD waves in magnetized flux tubes with a radial density inhomogeneity. Using the approximation that resistive and viscous processes are operative in thin layers surrounding the singularities of the MHD equations, they concluded that as the longitudinal wave number increases, the maximum amplitude of the body eigenmodes shifts away from the resonant layer and causes a decrease in damping rates.

An additional features of the flux tube is that of twist. There is observational evidence for the existence of twist in the solar atmosphere. The TRACE $171 \AA$ observations of a postflare loop system on 1999 confirmed the existence of the twisted loops in the corona (see Aschwanden 2005). Rotational movement along a loop observed by Chae et al. (2000) indicates the existence of twist. Aschwanden (2005) discusses observed noncoplanarity of loops which implies kinked field lines which, in turn, are also intimately related to twist. Besides, it is unfeasible that every flux tube within the solar atmosphere is entirely twist free in spite of the random continuous motions observed at the footpoints (see Erdélyi and Carter 2006). Following Aschwanden (2005), the magnetic twist parameter $B_{\phi} / B_{z}$ can be estimated as $B_{\phi} / B_{z}=\tan \theta$. Here $B_{\phi}$ and $B_{z}$ are the azimuthal and axial components of the magnetic field, respectively. Also $\theta$ is the shear angle between the untwisted and the twisted field line. The geometric shear angle $\theta$, can observationally be measured in twisted coronal loops.

Bennett, Roberts, and Narain (1999) examined the influence of magnetic twist on the modes of oscillations of a magnetic flux tube. They found that twist introduces an infinite band of body modes. Erdélyi and Fedun (2006) studied the wave propagation in a twisted cylindrical magnetic flux tube embedded in an incompressible but also magnetically twisted plasma. They found that increasing the external magnetic twist from 0 to 0.3 caused an increase in the normalized periods of sausage MHD waves approximately by $1-2 \%$. Erdélyi and Fedun (2007) extended the work of Erdélyi and Fedun (2006) to the case of compressible plasma. They found that increasing the internal twist may result in about up to $3-5 \%$ changes in periods for sausage modes.

Erdélyi and Carter (2006) studied the propagation of MHD waves in a fully magnetically twisted flux tube consisting of a core, annulus and external region. They investigated their analysis by considering magnetic twist just in the annulus, the internal and external regions having straight magnetic field. Two modes of oscillations occur in this configuration; surface and hybrid modes. They found that when the magnetic twist is increased the hybrid modes cover a wide range of phase speeds, centered around the annulus, longitudinal Alfvén speed for 
the sausage modes.

Carter and Erdélyi (2007) investigated the oscillations of a magnetic flux tube configuration consisting of a core, annulus and external region each with straight distinct magnetic field in an incompressible medium. They found that there are two surface modes arising for both the sausage and kink modes for the annulus-core model where the monolithic tube has solely one surface mode for the incompressible case. Also they showed that the existence and width of an annulus layer has an effect on the phase speeds and periods.

Carter and Erdélyi (2008) used the model introduced by Erdélyi and Carter (2006) to include the kink modes. They found for the set of kink body modes, the twist increases the phase speeds of the modes. Also they showed that there are two surface modes for the twisted shell configuration, one due to each surface, where one mode is trapped by the inner tube, the other by the annulus itself.

Mikhalyaev and Solov'ev (2005) investigated the MHD waves in a double magnetic flux tube embedded in a uniform external magnetic field. The tube consists of a dense hot cylindrical core surrounded by a co-axial shell. They found two slow and two fast magnetosonic modes can exist in the thin double tube.

Verwichte et al. (2004), using the observations of TRACE, detected multimode oscillations for the first time. They found that two loops are oscillating in both the fundamental and the first-overtone standing kink modes. According to the theory of MHD waves, for uniform loops the ratio of the period of the fundamental to the period of the first overtone is exactly 2 , but the ratios found by Verwichte et al. (2004) are $1.81 \pm 0.25$ and $1.64 \pm 0.23$. However, these values were corrected with the improvement of the observational error bars to $1.82 \pm 0.08$ and $1.58 \pm 0.06$, respectively, by Van Doorsselaere, Nakariakov, and Verwichte (2007). Also Verth, Erdélyi, and Jess (2008) added some further corrections by considering the very important effects of loop expansion and estimated a period ratio of 1.54. All these values clearly differ from 2. This may be caused by different factors such as the effects of curvature (see e.g. Van Doorsselaere et al. 2004b), leakage (see De Pontieu, Martens, and Hudson 2001), density stratification in the loops (see e.g. Andries et al. 2005; Erdélyi and Verth 2007; Karami and Asvar 2007; Safari, Nasiri, and Sobouti 2007; Karami, Nasiri, and Amiri 2009), magnetic field expansion (see Verth and Erdélyi 2008; Ruderman, Verth, and Erdélyi 2008; Verth et al. 2008) and magnetic twist (see e.g. Erdélyi and Fedun 2006, 2007; Erdélyi and Carter 2006; Karami and Barin 2009).

Karami and Barin (2009) studied both the oscillations and damping of standing MHD surface and hybrid waves in coronal loops in presence of twisted magnetic field. They considered a straight cylindrical incompressible flux tube with magnetic twist just in the annulus and straight magnetic field in the internal and external regions. They showed that both the frequencies and damping rates of both the kink and fluting modes increase when the twist parameter increases. They obtained that the period ratio $P_{1} / P_{2}$ of the fundamental and first-overtone for both the kink and fluting surface modes are lower than 2 (for untwisted loop) in presence of the twisted magnetic field. Ruderman (2007) studied nonaxisymmetric oscillations of thin twisted magnetic tubes in a zero-beta plasma by taking into account the longitudinal density stratification. Using the asymptotic analysis, he showed that the eigenmodes and eigenfrequencies of the kink and fluting oscillations are described by a classical Sturm-Liouville problem for a second-order ordinary differential equation. He also concluded that the results concerning nonaxisymmetric waves in twisted magnetic tubes obtained by Bennett et al. (1999) for incompressible plasmas can be applied to global nonaxisymmetric waves, i.e. kink and fluting modes, in coronal loops despite that the coronal plasma is a low-beta plasma. This conclusion was also in a good agreement with Erdélyi and Fedun (2007).

In the present work, our aim is to investigate the effect of twisted magnetic field on the 
resonant absorption of standing MHD waves in the coronal loops to justify the rapid damping of oscillations and deviation of the period ratio $P_{1} / P_{2}$ from 2 observed by TRACE. This paper is organized as follows. In Section 2 we derive the equations of motion, introduce the relevant connection formulae and obtain the dispersion relation. In Section 3 we give numerical results. Section 4 is devoted to conclusions.

\section{Equations of Motion}

The linearized MHD equations for an incompressible plasma are

$$
\begin{gathered}
\frac{\partial \delta \mathbf{v}}{\partial t}=-\frac{\nabla \delta p}{\rho}+\frac{1}{4 \pi \rho}\{(\nabla \times \delta \mathbf{B}) \times \mathbf{B}+(\nabla \times \mathbf{B}) \times \delta \mathbf{B}\}+\frac{\nu}{\rho} \nabla^{2} \delta \mathbf{v} \\
\frac{\partial \delta \mathbf{B}}{\partial t}=\nabla \times(\delta \mathbf{v} \times \mathbf{B})+\frac{c^{2}}{4 \pi \sigma} \nabla^{2} \delta \mathbf{B} \\
\nabla \cdot \delta \mathbf{v}=0,
\end{gathered}
$$

where $\delta \mathbf{v}, \delta \mathbf{B}$ and $\delta p$ are the Eulerian perturbations in the velocity, magnetic field and thermal pressure, respectively; $\rho, \sigma, \nu$ and $c$ are the mass density, the electrical conductivity, the viscosity and the speed of light, respectively. In the momentum Eq. (1) like Ruderman and Roberts (2002) we write the viscous force in a simplified form $\nu \nabla^{2} \delta \mathbf{v}$ instead of the classical Braginskii's expression for the viscosity tensor in a magnetized plasma (Braginskii 1965). Because according to Ruderman and Roberts (2002), under typical coronal conditions, the coefficient of the shear viscosity is at least 10 orders of magnitude smaller than that of the compressional viscosity. However, in the problem of oscillations of coronal loops, dissipation is only important in an Alfvénic dissipative layer embracing an ideal resonant magnetic surface. Numerical studies by Ofman et al. (1994), and Erdélyi and Goossens (1994, 1995) have shown that in Alfvénic dissipative layers only the shear viscosity is significant, all other terms in Braginskii's tensorial expression being neglected. Equation (3) satisfies the incompressibility condition. The assumption of incompressibility reduces the direct full applicability to coronal loops except for kink modes observed by the TRACE as they are highly incompressible perturbations (see Carter and Erdélyi 2007). Recently Goossens et al. (2009) showed that in the thin tube approximation neglecting contributions proportional to $\left(k_{z} R\right)^{2}$ then the frequency of the kink wave and its damping due to resonant absorption are the same in the three cases including a compressible pressureless plasma, an incompressible plasma and a compressible plasma which allows for MHD radiation. This is expected as kink modes are linearly incompressible. Note that here the energy equation is absent. Because for incompressible plasmas when sound speed (or adiabatic gas index) goes to infinity then the energy equation is decoupled from the system of linearized MHD equations.

The simplifying assumptions are as follows.

- The background magnetic field is assumed to be

$$
\mathbf{B}=\left(0, A r, B_{z}\right)
$$

where $A, B_{z}$ are constant and the magnetic field is uniformly twisted inside and outside the tube (see Fig. 1). This is unphysical as $r \rightarrow \infty$, and caution has to be exercised. 
Following Ruderman (2007), to satisfy the Shafranov-Kruskal stability criterion we assume that the azimuthal component of magnetic field is smaller than the axial component. This assumption is also compatible with observed weakly twisted coronal loops (see Erdélyi and Fedun 2006). The stability of a twisted magnetic flux tube has been studied in some details by Bennett et al. (1999), Ruderman (2007), and Carter and Erdélyi (2008). Note that the choice of $B_{\phi}=A r$ implies that there is a constant longitudinal current density along the flux tube.

- From Safari et al. (2006) and Karami et al. (2009), the density profile is assumed to be

$$
\rho(r)=\left\{\begin{array}{cc}
\rho_{\mathrm{i}}, & \left(r<R_{1}\right), \\
{\left[\frac{\rho_{\mathrm{i}}-\rho_{\mathrm{e}}}{R-R_{1}}\right](R-r)+\rho_{\mathrm{e}},} & \left(R_{1}<r<R\right), \\
\rho_{\mathrm{e}}, & (r>R),
\end{array}\right.
$$

where $R$ denotes the radius of the tube and $R_{1}<R$ is the radius at which the resonant absorption occurs. Also $\rho_{\mathrm{i}}$ and $\rho_{\mathrm{e}}$ are the interior and exterior constant densities of the tube, respectively (see Fig. 1). Note that for resonant absorption a number of density profiles have been considered. For instance, Ofman et al. $(1994,1995)$ have taken $\rho(r)=$ $\rho_{r}+\left(1-\rho_{r}\right) e^{-r^{4}}$ with $\rho_{r}=\rho_{\mathrm{e}} / \rho_{\mathrm{i}}$. Here since we use the thin boundary approximation, we assume that the inhomogeneous layer and the resonance layer coincide (see Goossens et al. 2009). Therefore any well behavior function of the density like that selected by Ofman et al. $(1994,1995)$ in the resonance layer, i.e. the region $R_{1}<r<R$ where the singularity occurs, can be approximated with a linearly varying profile.

- The equilibrium condition, i.e. radial force balance equation $\frac{\mathrm{d}}{\mathrm{d} r}\left[p(r)+\frac{B^{2}(r)}{8 \pi}\right]=-\frac{B_{\phi}^{2}(r)}{4 \pi r}$, gives the equilibrium plasma pressure $p(r)$ as

$$
p(r)=p_{0}-\frac{A^{2} r^{2}}{4 \pi}
$$

where $p_{0}$ is the plasma pressure at the center of the tube. Since we have no discontinuity in the background magnetic field, then the thermal pressure remains continuous across $r=R$ (see Fig. 1).

- Tube geometry is a circular with cylindrical coordinates, $(r, \phi, z)$.

- There is no initial steady flow over the tube.

- Viscous and resistive coefficients, $\nu$ and $\sigma$ respectively, are constant.

- $t$ -,$\phi$ - and $z$-dependence for any of the components $\delta \mathbf{v}$ and $\delta \mathbf{B}$ is $\exp \left\{\mathrm{i}\left(m \phi+k_{z} z-\omega t\right)\right\}$. Here $k_{z}=l \pi / L, L$ is the length of the tube, and $l=(1,2, \cdots), m=(0,1,2, \cdots)$ are the longitudinal and azimuthal mode numbers, respectively.

In the remainder of this section the following steps are taken: a) in $r<R_{1}$ and $r>R$, dissipative terms are neglected. Solutions of Eqs. (1) and (2) are obtained as in Bennett et al. (1999), Erdélyi and Carter (2006), and Karami and Barin (2009); b) in $R_{1}<r<R$, within which the resonant layer resides, we use the thin boundary (TB) approximation in which we assume that the inhomogeneous layer and the dissipative layer coincide (see Goossens et al. 2009). This enables us to avoid solving the non-ideal MHD equations in the inhomogeneous layer. The jumps across the resonant layer are found by the prescriptions of Sakurai et al. (1991a); c) substituting the solutions (a) in jump conditions (b) gives an analytical expression for a dispersion relation to be solved for the frequencies and the damping rates. 


\subsection{Interior and Exterior Solutions}

Following Bennett et al. (1999), Erdélyi and Carter (2006), and Karami and Barin (2009), in the absence of dissipations, taking time derivative of Eq. (2) and substituting for $\partial \delta \mathbf{v} / \partial t$ from Eq. (1), the resulting equation yields to Bessel's equation for the Eulerian perturbation in total pressure $\delta P_{\mathrm{T}}$ as

$$
\left[\frac{\mathrm{d}^{2}}{\mathrm{~d} r^{2}}+\frac{1}{r} \frac{\mathrm{d}}{\mathrm{d} r}-\left(\frac{m^{2}}{r^{2}}+m_{\mathrm{i}}^{2}\right)\right] \delta P_{\mathrm{T}}=0
$$

where

$$
\delta P_{\mathrm{T}}=\delta p+\frac{\mathbf{B} \cdot \delta \mathbf{B}}{4 \pi}
$$

and

$$
\begin{gathered}
m_{\mathrm{i}}^{2}=k_{z}^{2}\left[1-\frac{A^{2} \omega_{A_{\mathrm{i}}}^{2}}{\pi \rho_{\mathrm{i}}\left(\omega^{2}-\omega_{A_{\mathrm{i}}}^{2}\right)^{2}}\right], \\
\omega_{A_{\mathrm{i}}}=\frac{1}{\sqrt{4 \pi \rho_{\mathrm{i}}}}\left(m A+k_{z} B_{z}\right) .
\end{gathered}
$$

Note that Eq. (4) is valid only outside the inhomogeneous layer. Equation (4) is same as the result exactly derived by Bennett et al. (1999), and Erdélyi and Carter (2006). Also Eq. (4) in its general form for a compressible plasma was derived by Erdélyi and Fedun (2007). Note that subscripts $i$ (which correspond to the internal region) are replaced by $e$ corresponding to the external region. Solutions of Eq. (4) for the interior region $\left(r<R_{1}\right)$ are:

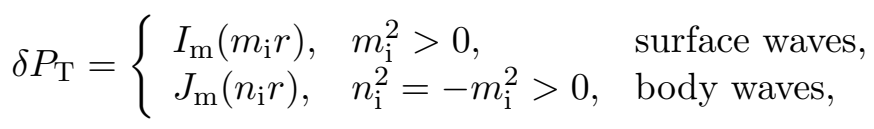

where $J_{\mathrm{m}}$ and $I_{\mathrm{m}}$ are Bessel and modified Bessel functions of the first kind, respectively. In the exterior region $(r>R)$, the waves should be evanescent. Solutions are

$$
\delta P_{\mathrm{T}}=K_{\mathrm{m}}\left(m_{\mathrm{e}} r\right), \quad m_{\mathrm{e}}^{2}>0,
$$

where $K_{\mathrm{m}}$ is the modified Bessel function of the second kind. One must note that from Eq. (6), solutions (9) are satisfied only for the frequencies $\omega^{2}>\omega_{A_{\mathrm{e}}}^{2}+\frac{A \omega_{A_{\mathrm{e}}}}{\sqrt{\pi \rho_{\mathrm{e}}}}$. Our numerical results in Sect. 3 confirms that this condition is hold for the obtained frequencies.

\subsection{Connection Formulae, Dispersion Relation and Damping}

According to the connection formulae given by Sakurai et al. (1991a), the jump across the boundary (resonance layer) for $\xi_{r}=-\delta v_{r} / \mathrm{i} \omega$ and $\delta P_{\mathrm{T}}$ is

$$
\begin{gathered}
{\left[\xi_{r}\right]=-\mathrm{i} \pi \frac{1}{|\Delta|} \frac{g_{B}}{\rho B^{2}} C_{\mathrm{A}},} \\
{\left[\delta P_{\mathrm{T}}\right]=-\mathrm{i} \pi \frac{1}{|\Delta|} \frac{A B_{z} F}{2 \pi \rho B^{2}} C_{\mathrm{A}},}
\end{gathered}
$$


where

$$
\begin{aligned}
C_{\mathrm{A}} & =g_{\mathrm{B}} \delta P_{T}-\frac{A B_{z} F}{2 \pi} \xi_{r}, \\
\Delta & =\left.\frac{\mathrm{d}}{\mathrm{d} r}\left(\omega^{2}-\omega_{\mathrm{A}}^{2}(r)\right)\right|_{r=r_{\mathrm{A}}}=\frac{\rho_{\mathrm{e}}-\rho_{\mathrm{i}}}{a \rho\left(r_{\mathrm{A}}\right)} \omega_{\mathrm{A}}^{2}\left(r_{\mathrm{A}}\right) \\
F & =m A+k_{z} B_{z}, \\
g_{\mathrm{B}} & =\frac{m B_{z}}{r}-k_{z} A r .
\end{aligned}
$$

Note that $a=R-R_{1}$ is the thickness of the inhomogeneous layer and $R_{1}<r_{\mathrm{A}}<R$ is the radius at which the singularity occurs. Davila (1987) showed that in the resonance absorption, however, the damping rate is independent of the dissipation coefficient values. But the resonance layer width scales as $\delta_{\mathrm{A}}=\left[\left|\frac{\omega}{\Delta}\right|\left(\frac{\nu}{\rho}+\frac{c^{2}}{4 \pi \sigma}\right)\right]^{1 / 3}$. Estimates for typical coronal values suggest that resonant layers have thicknesses from $0.3 \mathrm{~km}$ to $250 \mathrm{~km}$ (Davila 1987). According to Ofman et al. (1994), and Erdélyi and Goossens $(1994,1995)$ for resonant absorption the main contribution comes not from bulk viscosity but from shear viscosity. If we use the Reynolds $\mathcal{R}=\left(\frac{\mathrm{R}^{2} \rho_{\mathrm{i}}}{\nu}\right) /\left(\frac{2 \pi \mathrm{R}}{v_{A_{\mathrm{i}}}}\right)=560$ and Lundquist $S=\left(\frac{4 \pi \sigma \mathrm{R}^{2}}{c^{2}}\right) /\left(\frac{2 \pi \mathrm{R}}{v_{A_{\mathrm{i}}}}\right)=10^{4}$ numbers given by Ofman et al. (1994), and taking $L=109 \times 10^{3} \mathrm{~km}, R / L=0.01, a / R=0.08, \rho_{\mathrm{e}} / \rho_{\mathrm{i}}=0.1$, and interior Alfvén velocity $v_{A_{\mathrm{i}}}=2000$ $\mathrm{km} \mathrm{s}^{-1}$ for a typical coronal loop, then one can get $\delta_{\mathrm{A}} \simeq 85 \mathrm{~km}$ which is very close to the thickness of the inhomogeneous layer $a \simeq 87 \mathrm{~km}$. Therefore we can use the thin boundary approximation which assumes that the thickness of the resonance layer is the same as the inhomogeneous layer width (see Goossens et al. 2009).

Substituting the solutions of Eqs. (8)-(9) in jump conditions gives the dispersion relation as

$$
d_{0}(\tilde{\omega})+d_{1}(\tilde{\omega})=0
$$

where $\tilde{\omega}=\omega-\mathrm{i} \alpha, \alpha$ is damping rate and

$$
\begin{gathered}
d_{0}(\tilde{\omega})=\frac{m_{\mathrm{i}} R I_{\mathrm{m}}^{\prime}\left(m_{\mathrm{i}} R_{1}\right)}{I_{\mathrm{m}}\left(m_{\mathrm{i}} R_{1}\right)}+\frac{m F A R}{2 \pi R_{1} D_{\mathrm{i}}}-\frac{\lambda_{\mathrm{e}}}{\lambda_{\mathrm{i}}}\left(\frac{m_{\mathrm{e}} R K_{\mathrm{m}}^{\prime}\left(m_{\mathrm{e}} R\right)}{K_{\mathrm{m}}\left(m_{\mathrm{e}} R\right)}+\frac{m F A}{2 \pi D_{\mathrm{e}}}\right), \\
d_{1}(\tilde{\omega})=\frac{\mathrm{i} \pi}{|\Delta| \rho\left(r_{\mathrm{A}}\right) B^{2}\left(r_{\mathrm{A}}\right)} \frac{C_{\mathrm{A}}}{I_{\mathrm{m}}\left(m_{\mathrm{i}} R_{1}\right)}\left\{\frac{g_{\mathrm{B}} R}{\lambda_{\mathrm{i}}}+\frac{\lambda_{\mathrm{e}}}{\lambda_{\mathrm{i}}} \frac{F A B_{z}}{2 \pi}\left(\frac{m_{\mathrm{e}} R K_{\mathrm{m}}^{\prime}\left(m_{\mathrm{e}} R\right)}{K_{\mathrm{m}}\left(m_{\mathrm{e}} R\right)}+\frac{m F A}{2 \pi D_{\mathrm{e}}}\right)\right\},
\end{gathered}
$$

with

$$
\begin{aligned}
\lambda_{\mathrm{j}} & =\frac{D_{\mathrm{j}}}{D_{\mathrm{j}}^{2}-4(F A / 4 \pi)^{2}}, \\
D_{\mathrm{j}} & =\frac{F^{2}}{4 \pi}-\rho_{\mathrm{j}} \omega^{2},
\end{aligned}
$$

where (j) stands for (i) or (e). Also a prime on $I_{\mathrm{m}}$ and $K_{\mathrm{m}}$ indicates a derivative with respect to their appropriate arguments. The results for the body waves are the same as Eqs. (14)-(15), except that $I_{\mathrm{m}}$ is replaced by $J_{\mathrm{m}}$ everywhere.

Equations (10)-(11) show that when the twist is absent, i.e. $A=0$, the total Eulerian pressure will be continuous and only $\xi_{r}$ jumps across the boundary which is in agreement with Sakurai et al. (1991a).

For the surface modes, solving Eq. (13) yields one mode whose frequency exists between $\omega_{\mathrm{A}_{i}}$ and $\omega_{\mathrm{A}_{\mathrm{e}}}$. But for the body modes, there is an infinite band of body modes with the frequencies 
centered around $\omega_{\mathrm{A}_{\mathrm{i}}}$. The existence of an infinite set of body waves in the presence of twist has been already introduced by Bennett et al. (1999).

Note that the distinction between surface and body waves is not so important when we study the kink waves in slender magnetic tubes. Recently Goossens et al. (2009) concluded in the thin tube approximation, the kink MHD waves do not care about propagating (body wave) or evanescent (surface wave) behavior in the internal part of the flux tube. Although under coronal condition they are formally the body waves, they have typical properties of the surface waves. For example, the radial displacement takes its maximum value at the tube boundary. This is why Ruderman and Roberts (2002) suggested to call these waves the global kink waves. Also Erdélyi and Carter (2006) showed that under coronal conditions, for longer wavelength values the sausage surface mode only exists for smaller amounts of twist in the incompressible plasma.

The numerical solution of the dispersion relation (13) yields to frequencies $\omega_{n m l}$ and damping rates $\alpha_{n m l}$, which are characterized by a trio of wavenumbers $(n, m$ and $l)$ that actually count the number of nodes or antinodes along $r$-, $\phi$ - and $z$-directions, respectively.

\section{$3 \quad$ Numerical Results}

As typical parameters for a coronal loop, we assume $L=109 \times 10^{3} \mathrm{~km}, R / L=0.01, a / R=0.08$, $\rho_{\mathrm{e}} / \rho_{\mathrm{i}}=0.1, \rho_{\mathrm{i}}=2 \times 10^{-14} \mathrm{gr} \mathrm{cm}^{-3}, B_{z}=100 \mathrm{G}$. For such a loop one finds $v_{A_{\mathrm{i}}}=2000 \mathrm{~km} \mathrm{~s}^{-1}$, $\omega_{\mathrm{A}_{\mathrm{i}}}:=\frac{v_{\mathrm{A}_{\mathrm{i}}}}{L} \simeq 0.02 \mathrm{rad} \mathrm{s}^{-1}$.

The effects of twisted magnetic field on both the frequencies $\omega$ and damping rates $\alpha$ are calculated by numerical solution of the dispersion relation, i.e. Eq. (13). The results are displayed in Figs. 2 to 9. Figures 2 to 7 show the frequencies, damping rates and also the ratio of the oscillation frequency to the damping rate of the fundamental and first-overtone $l=1,2$ kink $(m=1)$ and fluting $(m=2,3)$ surface modes versus the twist parameter, $B_{\phi} / B_{z}:=\frac{A R}{B_{z}}$ for relative inhomogeneous layer width $a / R=0.08$. According to Carter and Erdélyi (2008), the kink $(m=1)$ speed is independent of the sound speed. Hence, the kink modes are highly incompressible, and this makes the limit of incompressibility be of great interest, not just for wave studies in the deeper part of the solar atmosphere (e.g. where the plasma-beta is high) but can also be directly applicable from the lower solar atmosphere to the corona. For all other modes, $(m \neq 1)$, compressibility may be necessary.

Figures 2 to 7 reveal that: i) the frequencies, the damping rates and the ratio $\omega / \alpha$ increase when the twist parameter increases. The result of $\omega$ is in good agreement with that obtained by Carter and Erdélyi (2008). Also the behavior of $\omega$ and $\alpha$ versus the twist parameter are in good concord with that obtained by Karami and Barin (2009). But, the numerical values of our damping rates for $m=1,2$ and 3 are six, seven and eight orders of magnitude, respectively, greater than those obtained by Karami and Barin (2009). This is due to existence of the resonant absorption which is absent in their work. Note that the dissipative processes in Karami and Barin (2009) depend on the Reynolds $\mathcal{R}$ and Lundquist $S$ numbers. They obtained the damping rates with $\mathcal{R}=560$ and $S=10^{4}$ given by Ofman et al. (1994). But in the resonance absorption, the damping rate is independent of the dissipation coefficient values and only the resonance layer width depends on $\mathcal{R}$ and $S$ (see Davila 1987). ii) For $m=1$ with increasing $B_{\phi} / B_{z}$, the frequencies, damping rates and their ratio, for instance $\omega_{111}, \alpha_{111}$ and $\omega_{111} / \alpha_{111}$, increase $\simeq 8$-66, $\simeq 6$-50 and $\simeq 2-11$ percent, respectively, compared with an untwisted loop. iii) For a given $m$ and $B_{\phi} / B_{z}$, when the longitudinal mode number, $l$, increases, both the frequencies and damping rates increase. But the ratio $\omega / \alpha$ : for $m=1$, for the twists lower than 0.003 increases and then decreases; for $m=2$, always decreases; but for $m=3$, for the twists lower than 0.115 
decreases and then increases. iv) For a given $l$ and $B_{\phi} / B_{z}$, when the azimuthal mode number, $m$, increases, the frequencies and damping rates increase but the ratio $\omega / \alpha$ decreases.

Here in our calculations, the sausage modes $(m=0)$ are absent. Because following Edwin and Roberts (1983), and Roberts, Edwin, and Benz (1984), the sausage modes have a cut-off longitudinal wave number $l_{\mathrm{c}}$, and they are only expected in fat and dense loops. According to Aschwanden (2005) the longitudinal wave number cut-off $l_{\mathrm{c}}$ for sausage-mode oscillations is expressed as a requirement of the loop length-to-width ratio $L /(2 R)$ as a function of the density contrast $\rho_{\mathrm{e}} / \rho_{\mathrm{i}}$ between the external and internal loop densities. For instance, for a typical active region, loops which have a density contrast of the order of $\rho_{\mathrm{e}} / \rho_{\mathrm{i}} \approx 0.1-0.5$ would be required to have length-to-width ratios of $L /(2 R) \approx 1-2$.

The period ratio $P_{1} / P_{2}$ of the fundamental and first-overtone, $l=1,2$ modes of both the kink $(m=1)$, and fluting $(m=2,3)$ surface waves versus the twist parameter is plotted in Fig. 8. Figure shows that for both kink and fluting modes, the period ratio $P_{1} / P_{2}$ decreases when the twist parameter increases. For instance, $P_{1} / P_{2}$ decreases from 2 (for untwisted loop) and approaches below 1.6, 1.2 and 1.1 for $m=(1,2$ and 3), respectively, with increasing the twist parameter. Note that when the twist is zero, the diagrams of $P_{1} / P_{2}$ do not start exactly from 2. This may be caused by the radial structuring $\rho_{\mathrm{e}} \neq \rho_{\mathrm{i}}$. But for the selected thin tube with $R / L=0.01$, this departure is very small, $O\left(10^{-3}\right)$ for kink $(m=1)$ and $O\left(10^{-4}\right)$ for fluting $(m=2, m=3)$ modes, and does not show itself in the diagrams (see McEwan et al. 2006). Figure 8 clears that for kink modes $(m=1)$, the ratio $P_{1} / P_{2}$ is 1.821 when $B_{\phi} / B_{z}=0.0065$, and is 1.584 for $B_{\phi} / B_{z}=0.0212$. These are in good agreement with the period ratios observed by Van Doorsselaere et al. (2007), $1.82 \pm 0.08$ and $1.58 \pm 0.06$, respectively, deduced from the observations of TRACE. Van Doorsselaere et al. (2007) considered only the effect of density stratification and neglected the effect of loop expansion. It has been shown by Verth and Erdélyi (2008) that failure to take account of the flux tube expansion causes the density scale height to be overestimated by a factor of approximately 2. Verth et al. (2008) considered both effects of the longitudinal density stratification and magnetic field expansion and estimated the correct coronal density scale height. They reported a period ratio of 1.54 deduced from the observations of TRACE for the kink modes. Here in our numerical calculations, if we take $B_{\phi} / B_{z}=0.0255$ and $\rho_{\mathrm{e}} / \rho_{\mathrm{i}}=0.05$ then we can obtain the period ratio $P_{1} / P_{2}=1.539$ for the kink modes which is in good agreement with that reported by Verth et al. (2008). This shows that the magnetic twist can achieve deviations from 2 of the same order of magnitude as in the model of Verth et al. (2008) containing the longitudinal density stratification and magnetic field expansion. Note that for the kink modes, the only period ratio obtained by Karami and Barin (2009) was 1.882 with $B_{\phi} / B_{z}=0.0065$. Therefore considering the magnetic twist in presence of the resonant absorption not only improved the period ratio estimated by Karami and Barin (2009) but also justified another period ratio, 1.54, reported by Verth et al. (2008).

Figure 9 displays the frequency band width, $\Delta \omega$, including infinite set of the fundamental kink $(m=1)$ body modes versus the twist parameter. Figure 9 presents that $\Delta \omega$ increases when the twist parameter increases. This is in good agreement with the result obtained by Carter and Erdélyi (2008), and Karami and Barin (2009).

\section{Conclusions}

Resonant absorption of standing MHD surface and body waves in coronal loops in the presence of the twisted magnetic field is studied. To do this, a typical coronal loop is considered as a straight cylindrical incompressible flux tube with magnetic twist that undergoes a radial density structuring. Under the thin tube approximation, the thickness of the inhomogeneous layer is 
same as the resonance layer width. Hence, the radial structuring is assumed to be a linearly varying density profile in the inhomogeneous layer. Using the relevant connection formulae, the dispersion relation is obtained and solved numerically for obtaining both the frequencies and damping rates of the fundamental and first-overtone kink and fluting modes. Our numerical results show that

i) the frequencies and damping rates as well as the ratio of the oscillation frequency to the damping rate of both the kink $(m=1)$ and fluting $(m=2,3)$ surface waves increase when the twist parameter increases. For $m=1$ with increasing $B_{\phi} / B_{z}$, the ratio $\omega_{111} / \alpha_{111}$ changes from 39.3 to 43.5 which approximately one order of magnitude is greater than the ratio reported by Nakariakov et al. (1999), Wang and Solanki (2004), and Verwichte et al. (2004) deduced from the TRACE data;

ii) the period ratio $P_{1} / P_{2}$, for both the kink $(m=1)$ and fluting $(m=2,3)$ surface modes is lower than 2 (for an untwisted loop) in the presence of the twisted magnetic field. The result of $P_{1} / P_{2}=1.821,1.539$ for kink modes is in agreement with the TRACE observations;

iii) Frequency bandwidth of the fundamental kink $(m=1)$ body modes increase when the twist parameter increases.

Note that the main differences between the present work and the work of Karami and Barin (2009) can be summarized as follows: i) in our model, the magnetic field was assumed to be uniformly twisted inside and outside the tube. Whereas they considered only a uniformly twisted magnetic annulus inside the loop. ii) They considered the weak damping due to viscous and resistive dissipations which cannot justify the rapid damping of the coronal loops observed by Nakariakov et al. (1999), and Wang and Solanki (2004). In the case one includes the resonant absorption, the rapid damping can be justified. iii) Our model could justify the period ratios of kink modes reported by Van Doorsselaere et al. (2007), i.e. $P_{1} / P_{2}=1.82,1.58$, and Verth et al. (2008), i.e. $P_{1} / P_{2}=1.54$, whereas Karami and Barin (2009) only justified $P_{1} / P_{2}=1.82$.

\section{Acknowledgments}

The authors thank the unknown referee for very valuable comments. The authors also thank Dr. H. Safari for a number of useful discussions. This work was supported by the Department of Physics, University of Kurdistan, Sanandaj, Iran.

\section{References}

[1] Andries, J., Goossens, M., Hollweg, J.V., Arregui, I., Van Doorsselaere, T.: 2005, Astron. Astrophys. 430, 1109.

[2] Aschwanden, M.J.: 2005, Physics of the Solar Corona. Springer, Berlin.

[3] Aschwanden, M.J., Fletcher, L., Schrijver, C.J., Alexander, D.: 1999, Astrophys. J. 520, 880.

[4] Bennett, K., Roberts, B., Narain, U.: 1999, Solar Phys. 185, 41.

[5] Braginskii, S.I.: 1965, Rev. Plasma Phys. 1, 205.

[6] Carter, B.K., Erdélyi, R.: 2007, Astron. Astrophys. 475, 323.

[7] Carter, B.K., Erdélyi, R.: 2008, Astron. Astrophys. 481, 239. 
[8] Chae, J., Wang, H., Qiu, J., et al.: 2000, Astrophys. J. 533, 535.

[9] Davila, J.M.: 1987, Astrophys. J. 317, 514.

[10] De Pontieu, B., Martens, P.C.H., Hudson, H.S.: 2001, Astrophys. J. 558, 859.

[11] Edwin, P.M., Roberts, B.: 1983, Solar Phys. 88, 179.

[12] Erdélyi, R.: 2004, Astron. Geophys. 45, 34.

[13] Erdélyi, R.: 2005, Publications of the Astronomy Department of the Eötvös University 15, 7.

[14] Erdélyi, R., Ballai, I.: 2007, Astron. Nachr. 328, 726.

[15] Erdélyi, R., Carter, B.K.: 2006, Astron. Astrophys. 455, 361.

[16] Erdélyi, R., Fedun, V.: 2006, Solar Phys. 238, 41.

[17] Erdélyi, R., Fedun, V.: 2007, Solar Phys. 246, 101.

[18] Erdélyi, R., Goossens, M.: 1994, Astrophys. Space Sci. 213, 273.

[19] Erdélyi, R., Goossens, M.: 1995, Astron. Astrophys. 294, 575.

[20] Erdélyi, R., Goossens, M.: 1996, Astron. Astrophys. 313, 664.

[21] Erdélyi, R., Verth, G.: 2007, Astron. Astrophys. 462, 743.

[22] Goossens, M., Hollweg, J.V., Sakurai, T.: 1992, Solar Phys. 138, 233.

[23] Goossens, M., Ruderman, M.S., Hollweg, J.V.: 1995, Solar Phys. 157, 75.

[24] Goossens, M., Andries, J., Aschwanden, M.J.: 2002, Astron. Astrophys. 394, L39.

[25] Goossens, M., Terradas, J., Andries, J., Arregui, I., Ballester, J. L.: 2009, Astron. Astrophys. 503, 213.

[26] Grotrian, W.: 1939, Naturwissenschaften 27, 214.

[27] Hollweg, J.V.: 1987a, Astrophys. J. 312, 880.

[28] Hollweg, J.V.: 1987b, Astrophys. J. 320, 875.

[29] Hollweg, J.V.: 1991, in Mechanisms of Chromospheric and Coronal Heating, ed. by P. Ulmschneider et al. (Springer, Berlin), p. 423.

[30] Ionson, J.A.: 1978, Astrophys. J. 226, 650.

[31] Karami, K., Asvar, A.: 2007, Mon. Not. Roy. Astron. Soc. 381, 97.

[32] Karami, K., Barin, M.: 2009, Mon. Not. Roy. Astron. Soc. 394, 521.

[33] Karami, K., Nasiri, S., Amiri, S.: 2009, Mon. Not. Roy. Astron. Soc. 394, 1973.

[34] Klimchuk, J.A.: 2006, Solar Phys. 234, 41. 
[35] McEwan, M.P., Donnelly, G.R., Díaz, A.J., Roberts, B.: 2006, Astron. Astrophys. 460, 893.

[36] Mikhalyaev, B.B., Solov'ev, A.A.: 2005, Solar Phys. 227, 249.

[37] Nakariakov, V.M., Ofman, L., DeLuca, E.E., Roberts, B., Davila, J.M.: 1999, Science 285, 862.

[38] Narain, U., Ulmschneider, P.: 1996, Space Sci. Rev. 75, 453.

[39] Ofman, L., Davila, J.M., Steinolfson, R.S.: 1994, Astrophys. J. 421, 360.

[40] Ofman, L., Davila, J.M., Steinolfson, R.S.: 1995, Astrophys. J. 444, 471.

[41] Ofman, L., Aschwanden, M.J.: 2002, Astrophys. J. 576, L153.

[42] Poedts, S., Goossens, M., Kerner, W.: 1989, Solar Phys. 123, 83.

[43] Poedts S., Goossens M., Kerner W.: 1990, Astrophys. J. 360, 279.

[44] Rae, I.C., Roberts, B.: 1982, Mon. Not. Roy. Astron. Soc. 201, 1171.

[45] Roberts, B., Edwin, P. M., Benz, A. O.: 1984, Astrophys. J. 279, 857.

[46] Ruderman M.S.: 2007, Solar Phys. 246, 119.

[47] Ruderman, M.S., Roberts, B.: 2002, Astrophys. J. 577, 475.

[48] Ruderman, M.S., Verth, G., Erdélyi, R.: 2008, Astrophys. J. 686, 694.

[49] Safari, H., Nasiri, S., Karami, K., Sobouti, Y.: 2006, Astron. Astrophys. 448, 375.

[50] Safari, H., Nasiri, S., Sobouti, Y.: 2007, Astron. Astrophys. 470, 1111.

[51] Sakurai, T., Goossens, M., Hollweg, J.V.: 1991a, Solar Phys. 133, 227.

[52] Sakurai, T., Goossens, M., Hollweg, J.V.: 1991b, Solar Phys. 133, 247.

[53] Smith, P.D., Tsiklauri, D., Ruderman, M.S.: 2007, Astron. Astrophys. 475, 1111.

[54] Steinolfson, R.S., Davila, J.M.: 1993, Astrophys. J. 415, 354.

[55] Taroyan, Y., Erdélyi, R.: 2009, DOI 10.1007/s11214-009-9506-9.

[56] Van Doorsslaere, T., Andries, J., Poedts, S., Goossense, M.: 2004a, Astrophys. J. 606, 1223.

[57] Van Doorsselaere, T., Debosscher, A., Andries, J., Poedts, S.: 2004b, Astron. Astrophys. 424, 1065.

[58] Van Doorsslaere, T., Nakariakov, V.M., Verwichte, E.: 2007, Astron. Astrophys. 473, 959.

[59] Verth, G., Erdélyi, R.: 2008, Astron. Astrophys. 486, 1015.

[60] Verth, G., Erdélyi, R., Jess, D.B.: 2008, Astrophys. J. 687, L45.

[61] Verwichte, E., Nakariakov, V.M., Ofman, L., Deluca, E.E.: 2004, Solar Phys. 223, 77.

[62] Wang, T.J., Solanki, S.K.: 2004, Astron. Astrophys. 421, L33.

[63] Walsh, R.W., Ireland, J.: 2003, Astron. Astrophys. Rev. 12, 1. 

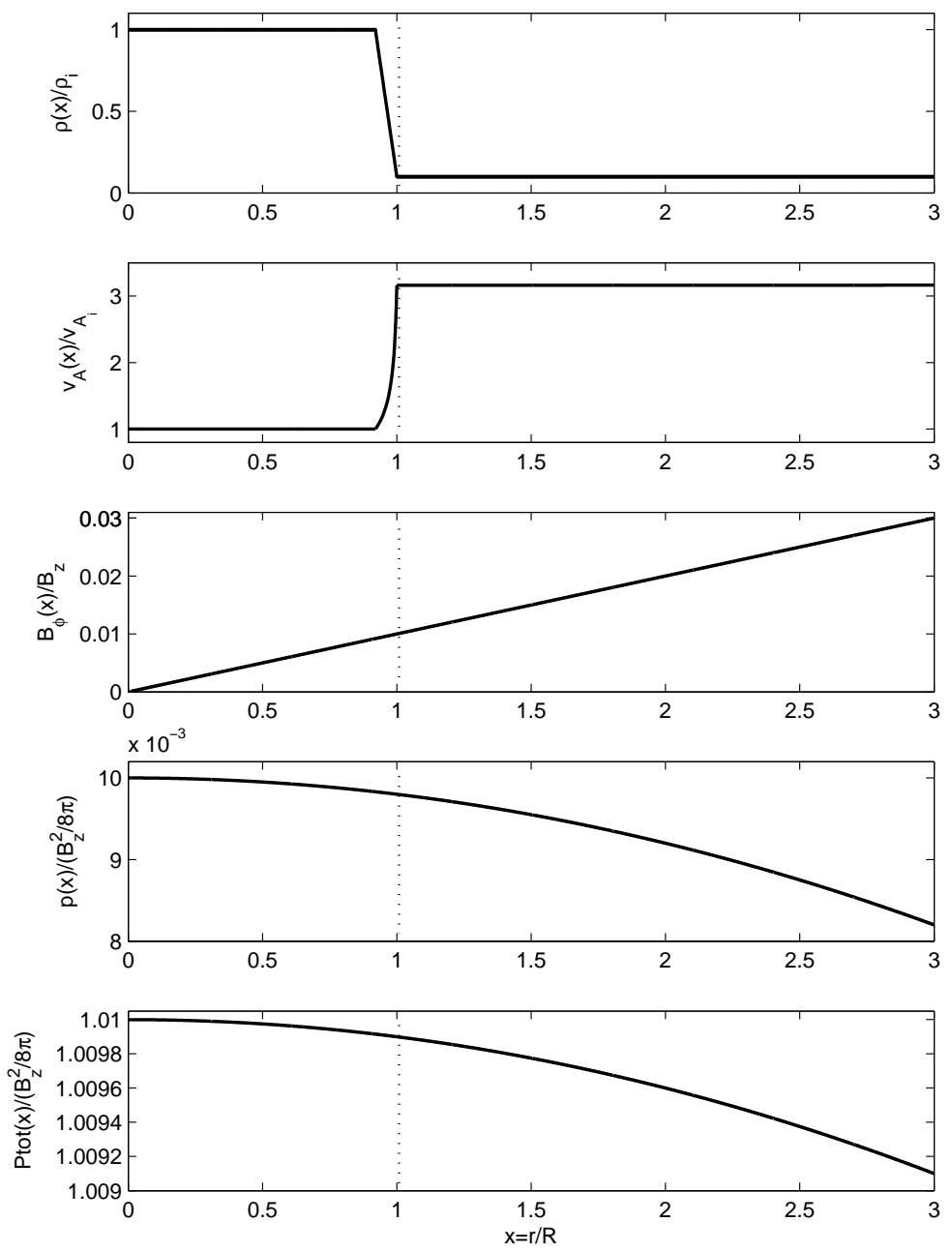

Figure 1: Dimensionless equilibrium quantities including density $\rho$, Alfvén velocity $v_{\mathrm{A}}$, azimuthal component of the uniformly twisted magnetic field $B_{\phi}$, thermal pressure $p$, and total pressure $p_{\text {tot }}=p+B^{2} /(8 \pi)$ against fractional radius $x=r / R$. Auxiliary parameters are $\rho_{\mathrm{e}} / \rho_{\mathrm{i}}=0.1$, $\frac{A R}{B_{z}}=0.01, \beta=\frac{p_{0}}{B_{z}^{2} /(8 \pi)}=0.01$. The parameter $\beta$ has been taken from Smith, Tsiklauri, and Ruderman (2007). 
Kink Surface Modes $(\mathrm{m}=1, \mathrm{l}=1)$
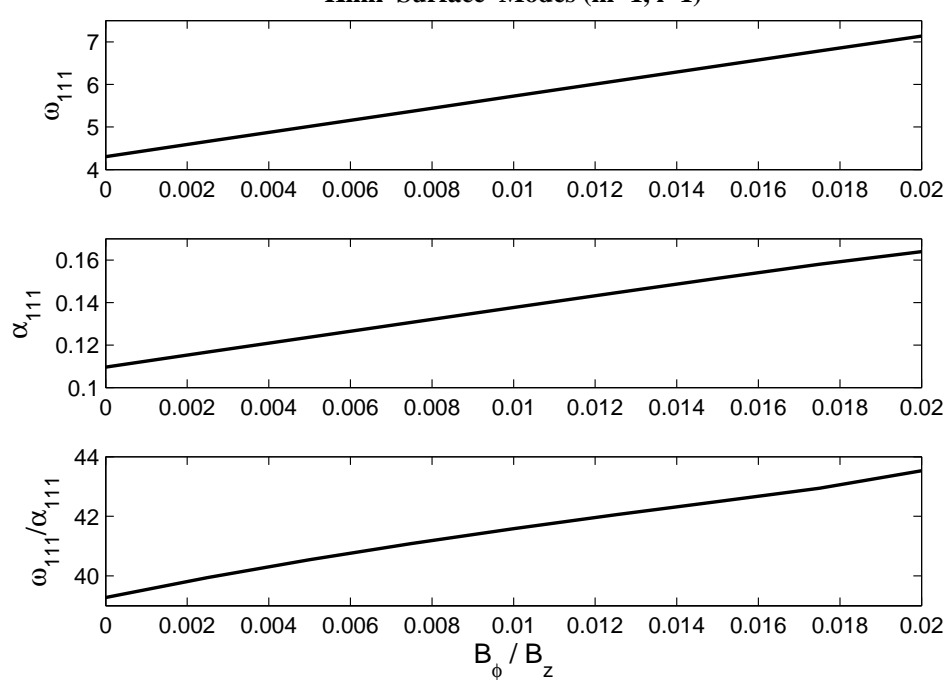

Figure 2: Frequency of the fundamental kink $(m=1)$ surface mode, its damping rate and the ratio of the oscillation frequency to the damping rate versus the twist parameter, $B_{\phi} / B_{z}$. The loop parameters are: $L=109 \times 10^{3} \mathrm{~km}, R / L=0.01, a / R=0.08, \rho_{\mathrm{e}} / \rho_{\mathrm{i}}=0.1, \rho_{\mathrm{i}}=2 \times 10^{-14}$ $\mathrm{gr} \mathrm{cm}^{-3}, B_{z}=100 \mathrm{G}$. Both frequencies and damping rates are in units of the interior Alfvén frequency, $\omega_{\mathrm{A}_{\mathrm{i}}} \simeq 0.02 \mathrm{rad} \mathrm{s}^{-1}$.
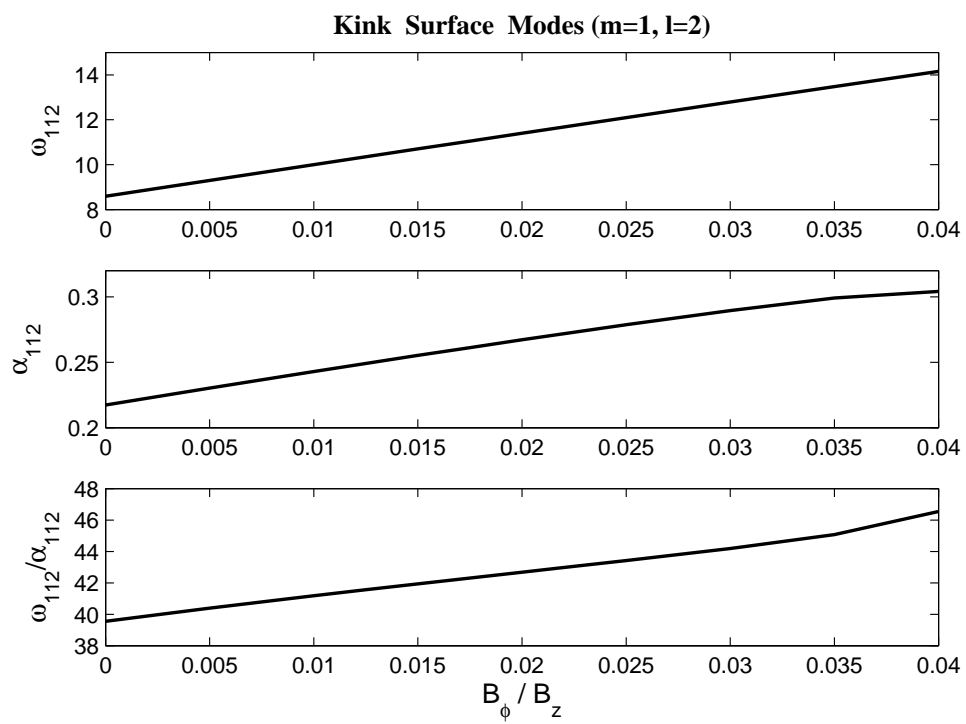

Figure 3: Same as Fig. 2, for the first-overtone kink $(m=1)$ surface modes. 

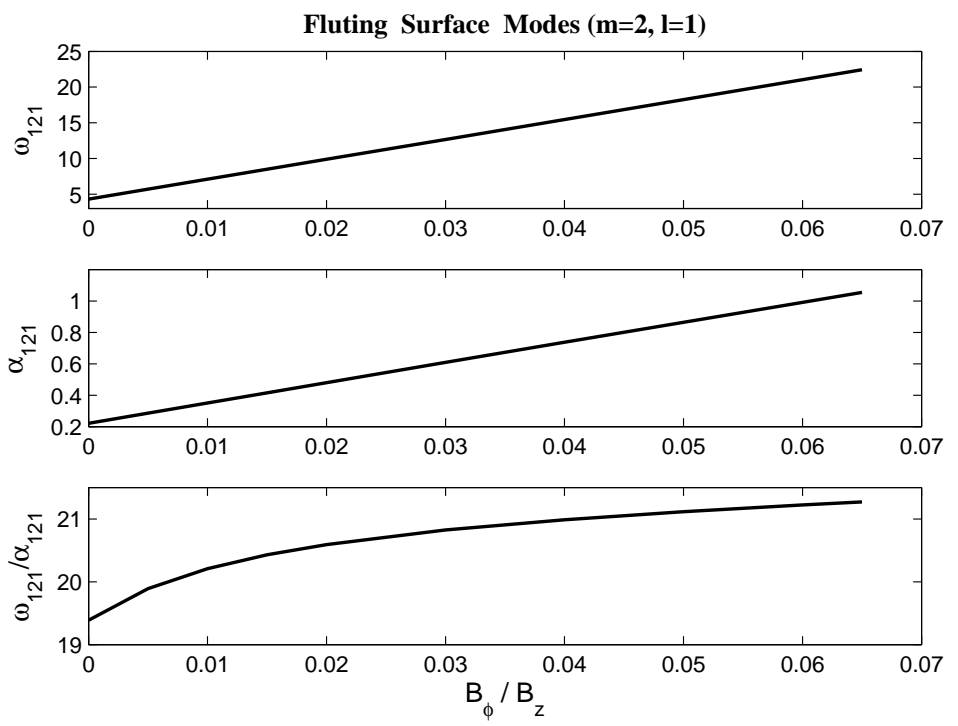

Figure 4: Same as Fig. 2, for the fundamental fluting $(m=2)$ surface modes.
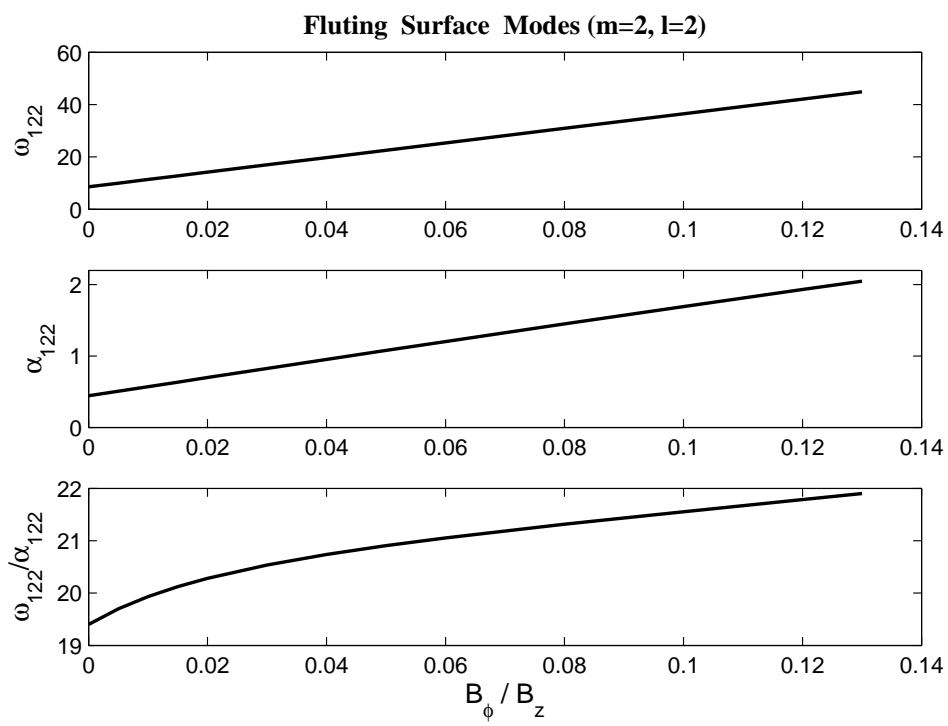

Figure 5: Same as Fig. 2, for the first-overtone fluting $(m=2)$ surface modes. 
Fluting Surface Modes $(\mathrm{m}=\mathbf{3}, \mathrm{l}=\mathbf{1})$
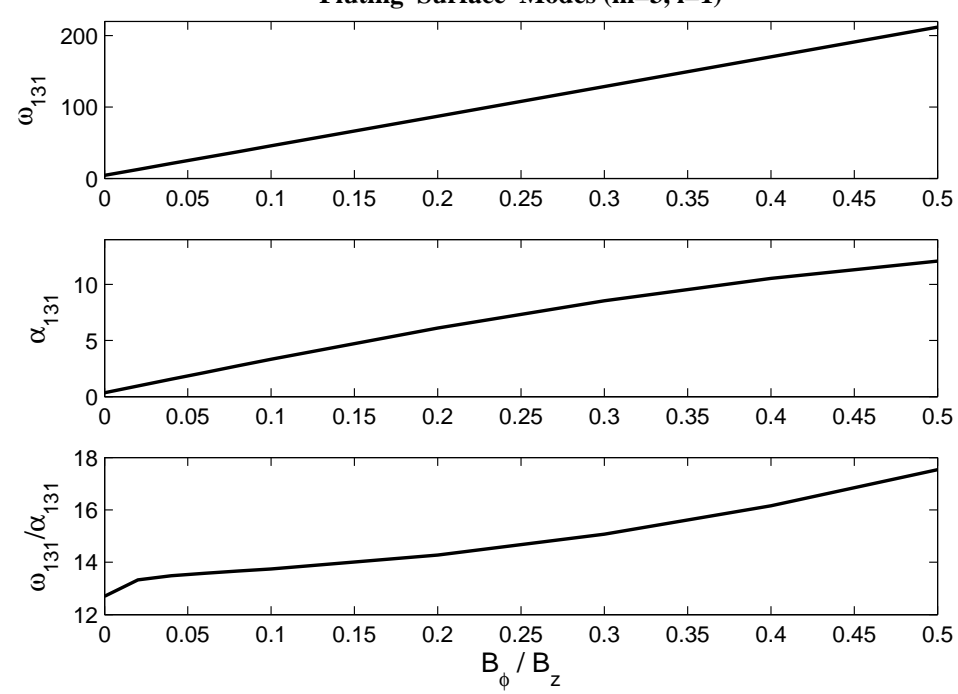

Figure 6: Same as Fig. 2, for the fundamental fluting $(m=3)$ surface modes.

Fluting Surface Modes $(\mathrm{m}=3, \mathrm{l}=2)$
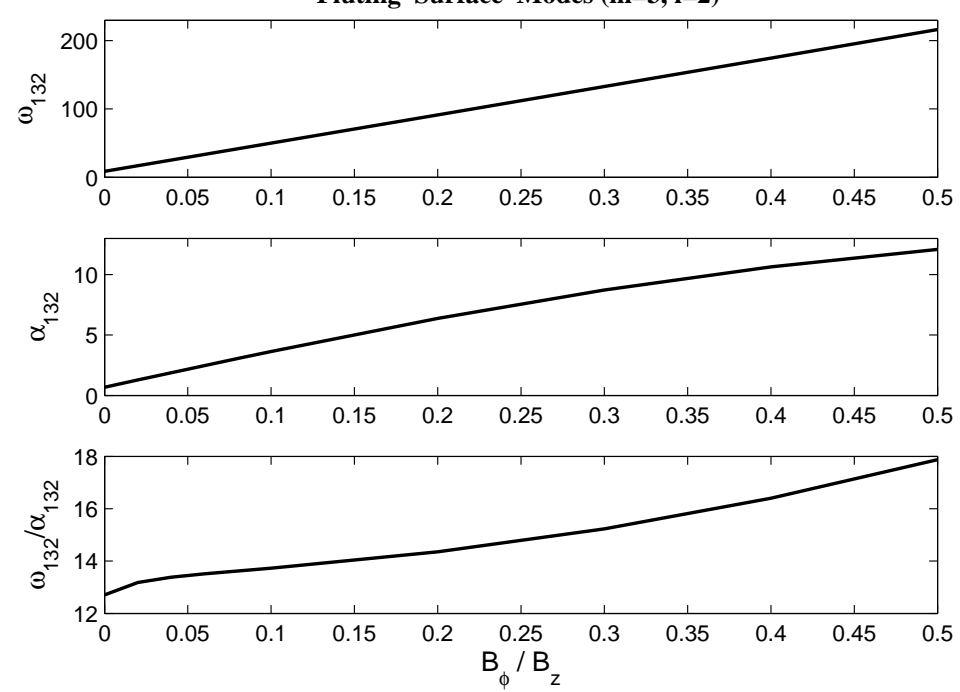

Figure 7: Same as Fig. 2, for the first-overtone fluting $(m=3)$ surface modes. 

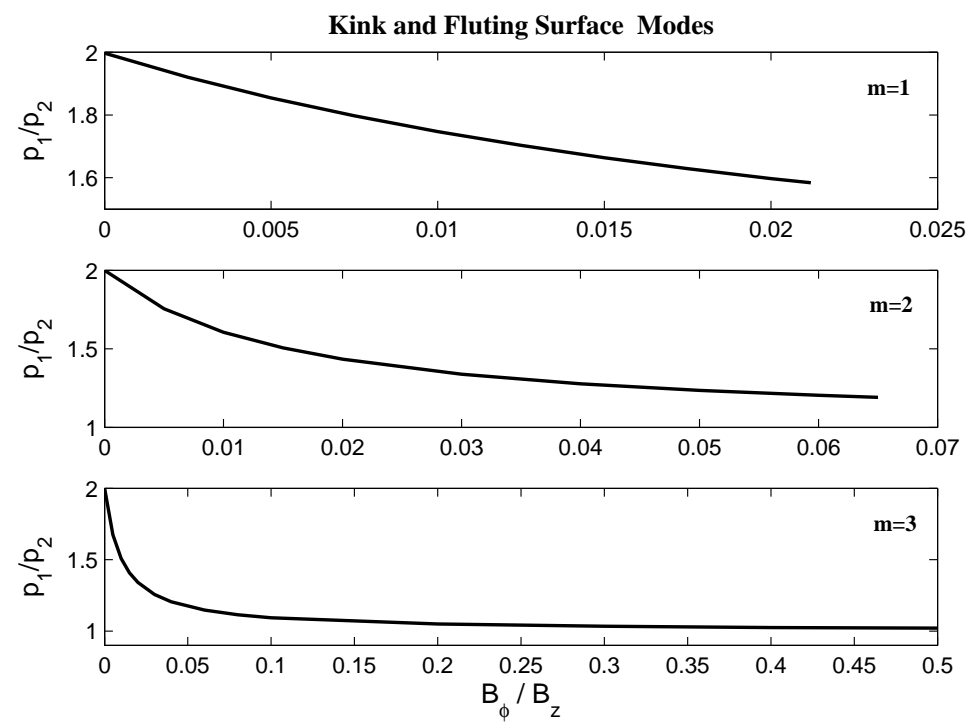

Figure 8: The period ratio $P_{1} / P_{2}$ of the fundamental and its first-overtone surface modes versus the twist parameter for kink $(m=1)$ and fluting $(m=2,3)$ modes. Auxiliary parameters as in Fig. 2.

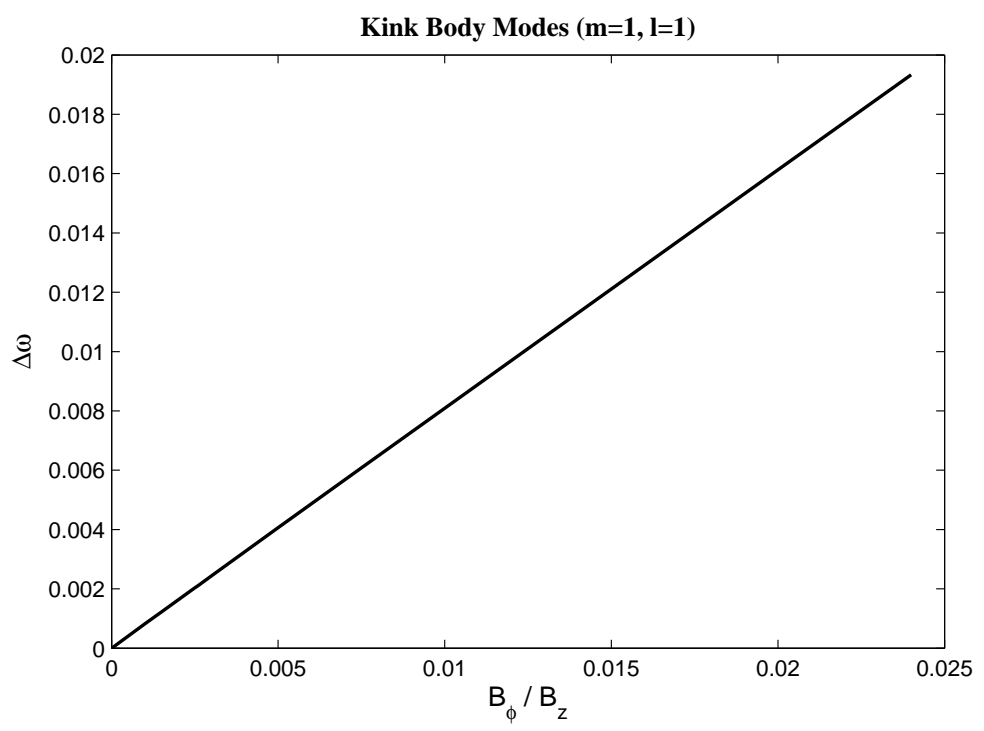

Figure 9: Frequency bandwidth of the fundamental kink $(m=1)$ body modes versus the twist parameter for the relative inhomogeneous layer width $a / R=0.08$. Auxiliary parameters as in Fig. 2. 